\title{
Cancerous 'floater': a lesson learned about tissue identity testing, endometrial cancer and microsatellite instability
}

\author{
Veerle Bossuyt ${ }^{1}$, Natalia Buza ${ }^{1}$, Nhu T Ngo ${ }^{1}$, Melissa A Much ${ }^{1}$, Maria C Asis ${ }^{2}$, \\ Peter E Schwartz ${ }^{2}$ and Pei Hui ${ }^{1,2}$ \\ ${ }^{1}$ Department of Pathology, Yale University School of Medicine, New Haven, CT, USA and ${ }^{2}$ Department of \\ Obstetrics, Gynecology and Reproductive Sciences, Yale University School of Medicine, New Haven, \\ CT, USA
}

\begin{abstract}
A 46-year-old woman presented with endometrial cells on a pap smear and underwent endometrial curettage. The specimen revealed secretory endometrium and a possible endometrial polyp. In addition, a single $4 \mathrm{~mm}$ fragment of well-differentiated adenocarcinoma was found. Tissue identity DNA genotyping was performed and the adenocarcinoma tissue fragment showed a drastically different allelic pattern from that of the background endometrium. To confirm tissue contamination, genotyping of three other tumor specimens-probable sources for a contaminant-was performed but failed to identify a match. Without confirmation of contamination, a second endometrial curettage was obtained from the patient, in which similar adenocarcinoma tissue was once again found. Further workup demonstrated that the patient had a microsatellite unstable (MSI) endometrial adenocarcinoma by immunohistochemistry and molecular testing. The patient subsequently underwent staging surgery, which revealed an early-stage, well-differentiated endometrioid adenocarcinoma. This case study illustrates an uncommon, yet important caveat of tissue identity testing by DNA genotyping, where MSI instability can significantly alter the allelic pattern of DNA polymorphisms in the tumor genome, leading to erroneous conclusion regarding the tissue identity. Awareness of this phenomenon is crucial for a molecular pathologist to avoid interpretation errors of tissue identity testing in a cancer diagnostic workup. Modern Pathology (2013) 26, 1264-1269; doi:10.1038/modpathol.2013.63; published online 5 April 2013
\end{abstract}

Keywords: malignancy; MSI; tissue identity testing

Not uncommonly, a surgical pathologist faces challenges regarding interpretation of tissue fragments that morphologically do not fit to the specimen at hand, known as 'floaters'. ${ }^{1}$ This occurs as a result of carrying over tissue pieces from one case to another during specimen grossing, sectioning, embedding, microtome cutting, and even routine staining in an anatomic pathology laboratory. Although pathologists can easily resolve most of such cases on the basis of tissue types and other histological findings, it may pose significant interpretation difficulties at the microscope when the floater has the same tissue type or is a significant lesion. The most challenging scenario in dealing

Correspondence: Dr P Hui, MD, PhD, Department of Pathology, Yale University School of Medicine, P.O. Box 208023, 310 Cedar Street BML 250, New Haven, CT 06520-8023, USA.

E-mail: pei.hui@yale.edu

Received 5 January 2013; revised 16 February 2013; accepted 17 February 2013; published online 5 April 2013 with a possible floater is when the tissue in question consists of cancer that can be neither ignored nor admitted as the malignancy of the patient at hand with complete confidence. Such uncertainty generally will lead to a phone call to the submitting physician, with a recommendation to rebiopsy the patient. Therefore, when tissue contamination or mislabeling may result in a significant medical decision, for example, a diagnosis of malignancy, an error-proof confirmation is highly desirable. Tissue identity genotyping can offer tremendous help in resolving these issues. ${ }^{1,2}$ Although some popular commercial genotyping kits using short-tandem repeat (STR) polymorphism offer more than sufficient discriminating power, one rare but significant caveat of tissue identity testing of neoplastic tissue is the presence of microsatellite instable (MSI) tumors, in which genetic alterations may drastically change the number of STR repeat in the tumor genome, leading to unexpected allelic discordance between 
the tumor and the corresponding normal tissue, and therefore an erroneous interpretation. ${ }^{2}$ Among all cancer types, colorectal and endometrial cancers are commonly involved by MSI. We report our experience of cancer tissue identity testing for a 46-yearold woman who developed an MSI endometrial adenocarcinoma.

\section{Case description}

A 46-year-old thin healthy woman presented for her annual gynecologic visit with no complaints. A routine pap smear was performed, which showed presence of endometrial cells and no other abnormalities. When contacted with this finding, the patient explained that her periods had become increasingly heavy and associated with premenstrual spotting over the past few months. She reported no other significant prior personal or family history. The endometrial lining appeared normal on ultrasound. The patient did not want a biopsy because of concerns about pain. She underwent an endometrial curettage. Microscopic evaluation revealed secretorytype endometrium without definitive evidence of hyperplasia. Some fragments of endometrium showed altered stroma, irregular distribution of glands, and prominent blood vessels, suggesting a possible endometrial polyp. In addition, a single $4 \mathrm{~mm}$ fragment of well-differentiated adenocarcinoma was present (Figure 1a). The fragment appeared morphologically distinct, and physically separated from the rest of background secretory endometrium (rather than proliferative or hyperplastic endometrium). Although it remained possible that the patient had a primary endometrial adenocarcinoma or a primary tubal/ovarian carcinoma with 'drop' metastasis into the endometrial cavity, overall because of the unusual presentation on the slides and low suspicion for carcinoma clinically, the possibility of a tissue floater was seriously considered.

Genomic DNA was extracted from tissue sections of both the cancerous tissue floater and the patient's background endometrium. DNA genotyping analysis was performed by AmpFlSTR Identifiler PCR Amplification system (Applied Biosystems). The reaction consists of a short-tandem repeat (STR) multiplex PCR assay that amplifies 15 different tetranucleotide repeat loci in a single reaction producing short amplicons ranging from 100 to 350 base pairs. The assay tests 13 loci of the Combined DNA Index System (CODIS) plus 2 additional loci, D2S1338 and D19S433. Genomic DNA of $2.5 \mathrm{ng}$ was amplified in a $25-\mu$ l reaction containing $10.0 \mu \mathrm{l}$ of AmpFlSTR reaction mix, $5.0 \mu \mathrm{l}$ of primer mix, and $0.5 \mu \mathrm{l}$ AmpliTaq Gold DNA polymerase. Details of PCR reaction and capillary electrophoresis have been previously published. ${ }^{3}$ Tissue identity was determined by comparing the genotype of the cancerous tissue with that of background endometrium of the patient. The
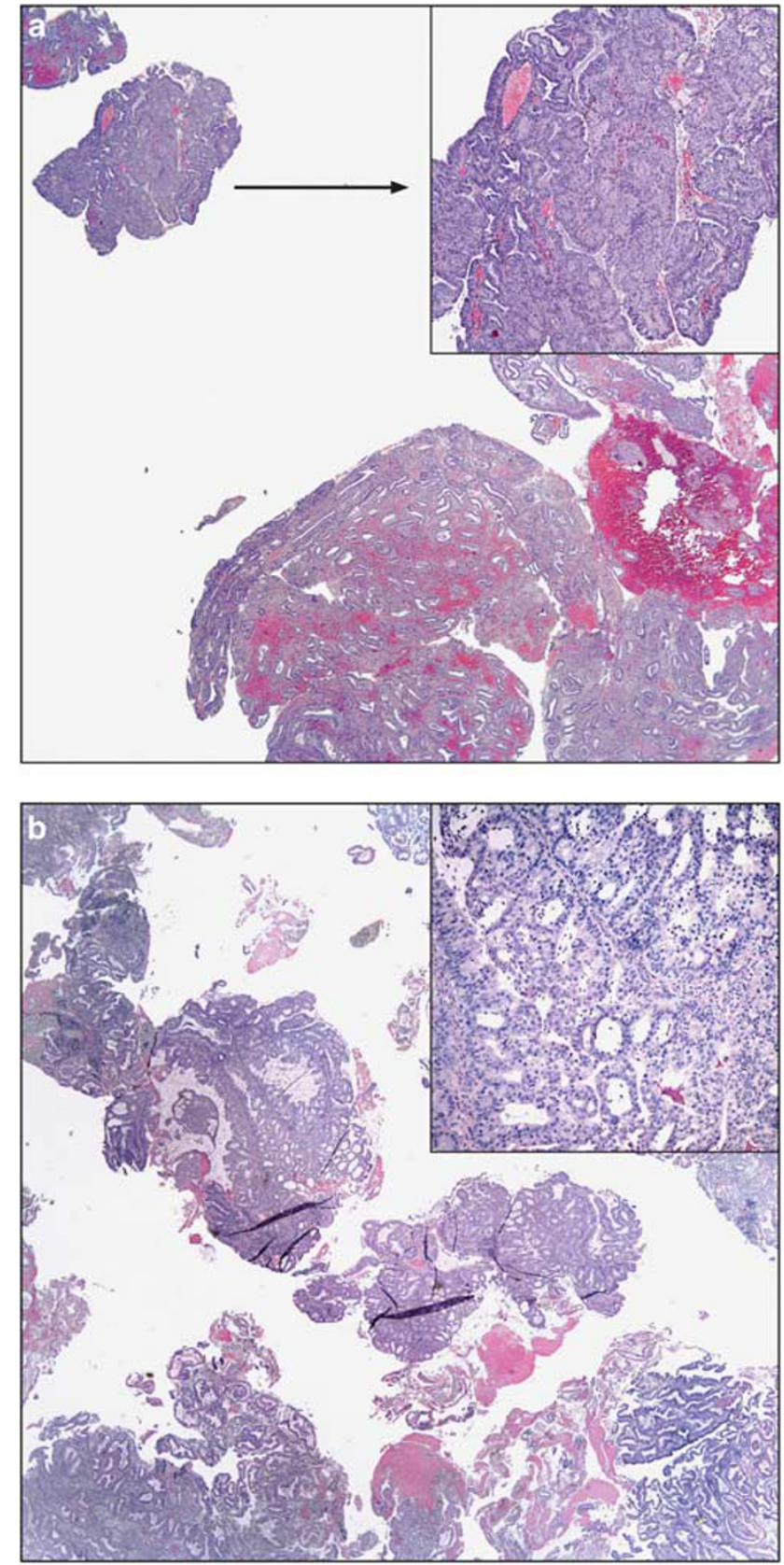

Figure 1 Low power H\&E of the patient's first endometrial curetting $(\mathbf{a}, \times 4)$. The majority of the specimen shows secretory endometrium with some fragments suggestive of an endometrial polyp. In the upper left corner a small fragment of endometrial adenocarcinoma is identified (insert, $\times 20$ ). Low power H\&E of the patient's second endometrial curetting $(\mathbf{b}, \times 4)$. Again, small fragments of endometrial adenocarcinoma (center and insert, $\times 20$ ) are identified in a background of secretory endometrium.

genotyping results showed a drastically different allelic pattern of the cancerous floater compared with the background endometrium (Figure 2).

In an attempt to confirm tissue contamination, identity testing of three other tumor specimens from three different patients was pursued, but failed to reveal the source of the 'contamination'. In the absence of an expected molecular confirmation, the 

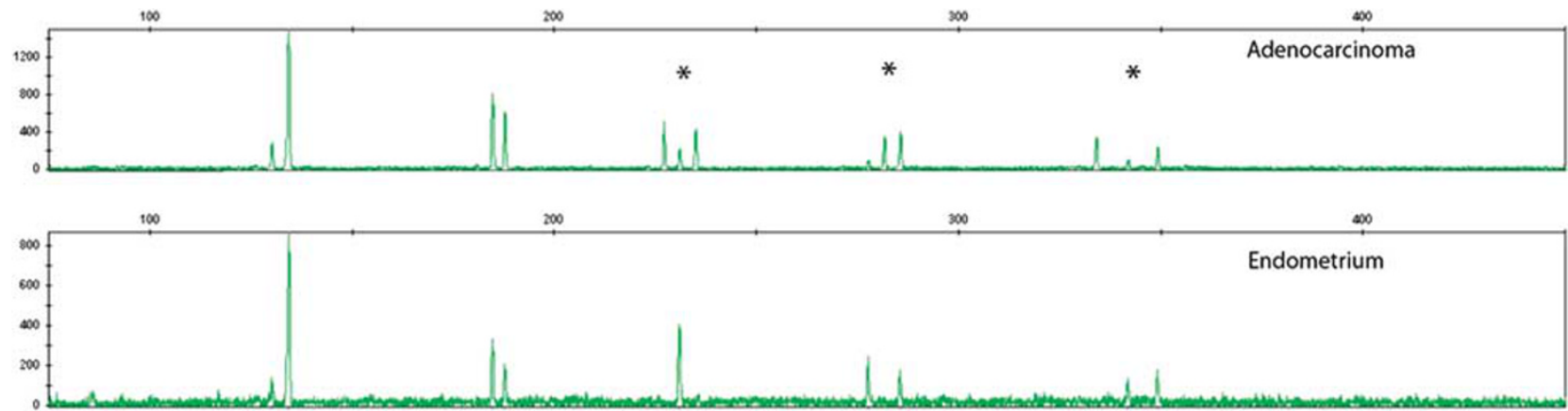

Figure 2 Tissue genotyping evaluation of the patient's first endometrial curetting (upper panel, cancerous tissue fragment and lower panel, normal secretory endometrium). Note the presence of different allelic patterns at three (indicated by ${ }^{*}$ ) of the five STR loci between the adenocarcinoma and the normal endometrium.

patient underwent a second endometrial curettage, in which fragments of adenocarcinoma of identical histology were 'surprisingly' found once again (Figure 1b). Furthermore, tissue genotyping also showed a discordant genetic profile similar to that of the first specimen (data not shown).

Further workup revealed that the patient has a microsatellite unstable endometrioid adenocarcinoma, by immunohistochemistry and MSI molecular testing. The tumor cells expressed $M L H-1$ and $P M S$-2 proteins by immunohistochemistry but did not express $M S H-2$ and $M S H-6$ proteins (Figure 3a). DNA samples from the tumor and normal endometrium were amplified by multiplex PCR for the standard five microsatellite markers: BAT 25, BAT 26, D2S123, D5S346, and D17S250. ${ }^{4}$ Microsatellite instability was detected at three of five microsatellite loci, indicating an MSI-high tumor (Figure 3b).

The patient subsequently underwent a staging surgery, which revealed a FIGO stage IA welldifferentiated endometrioid adenocarcinoma (FIGO grade 1). No myometrial invasion, lymphovascular invasion, lower uterine segment involvement, or endocervical involvement were seen. The background endometrium was in the secretory phase with focal complex hyperplasia. A single $0.6 \mathrm{~cm}$ leiomyoma was also present. The ovaries and fallopian tubes were uninvolved. Thirteen right pelvic, five left pelvic, and two para-aortic lymph nodes were negative for tumor. No adjuvant treatment was recommended. The patient was referred for genetic counseling because of the possibility of Lynch Syndrome. A detailed family history suggested the possibility of a PTEN mutation because of a remote family history of thyroid malignancy. However, no mutations of MSH2, MSH6, or PTEN were identified in subsequent evaluations. The patient is well and has negative follow-up pap smears 1 year later.

\section{Discussion}

Genotyping provides a measurement of the genetic variation between members of a species, and there- fore can be used to identify the source of DNA or genomic haploid set in a cell or tissue sample. Tissue identity testing by DNA genotyping is an extension from its initial application in forensic identity and parentage testing. Specimen mislabeling, tissue mix-up, and cross-contamination are not infrequent problems encountered in the daily practice of surgical pathology, yet carry far-reaching diagnostic and legal implications. Specimen mislabeling or mix-up may occur at the time of tissue collection in the submitting physician's office, the operating room, or during tissue processing in the surgical pathology suite. Histological contamination or floaters may happen during specimen grossing, embedding, sectioning, or histological staining. When mislabeling and particularly mix-up occur, tissue identity may not be resolved by routine laboratory investigations. Although malignant tissue floaters may be suspected by a surgical pathologist, seldom is such a case ignored with complete confidence, and a request for a repeat biopsy from the patient is frequently made. Therefore, when tissue contamination or mislabeling may result in a significant medical decision, for example, a diagnosis of malignancy, an error-proof confirmation is highly desirable. Human tissue identity testing based on DNA polymorphisms can offer tremendous help in resolving these issues. ${ }^{1,2}$

Human identity testing based on DNA shorttandem repeat (STR) polymorphisms has a powerful application in the forensic world. With recent commercial availability of cost-effective assays, PCR-based STR genotyping evaluation has been found to have significant applications in diagnostic pathology, including specimen identity testing, bone marrow transplant chimerism/engraftment evaluation, and recently the diagnosis and subclassification of hydatidiform moles. Short-tandem repeats (STR) are repetitive DNA sequences of 2-7 nucleotides, which are highly polymorphic, genetically stable and prevalent in the non-coding regions of the human genome. ${ }^{5}$ By identification of the number of the STR at specific loci, a genetic profile of an individual or a cell can be ascertained to distinguish one from another. The assay is highly 
accurate and reproducible when testing genetically stable cells or tissues. However, the presence of genetic instability, particularly microsatellite instability (MSI) in some tumors, may significantly complicate the application of the assay. The current case fully illustrates such a caveat, being unaware of which may lead to potentially devastating medical and legal consequences.

DNA mismatch repair genes (MMR) are responsible for repairing single-nucleotide mismatches, insertion, and deletion mutations. ${ }^{6}$ Microsatellites are STR sequences that are susceptible to acquiring mutations when MMR function is impaired. Tumor cells with defective MMR demonstrate an altered number of microsatellite nucleotide repeats when compared with paired normal tissue, a finding referred to as microsatellite instability. In turn, MSI will significantly affect the status of STR loci interrogated by DNA identity testing. As illustrated in our case, the presence of MSI tumor may significantly alter the wild-type allelic polymorphism in the patient, leading to interpretation

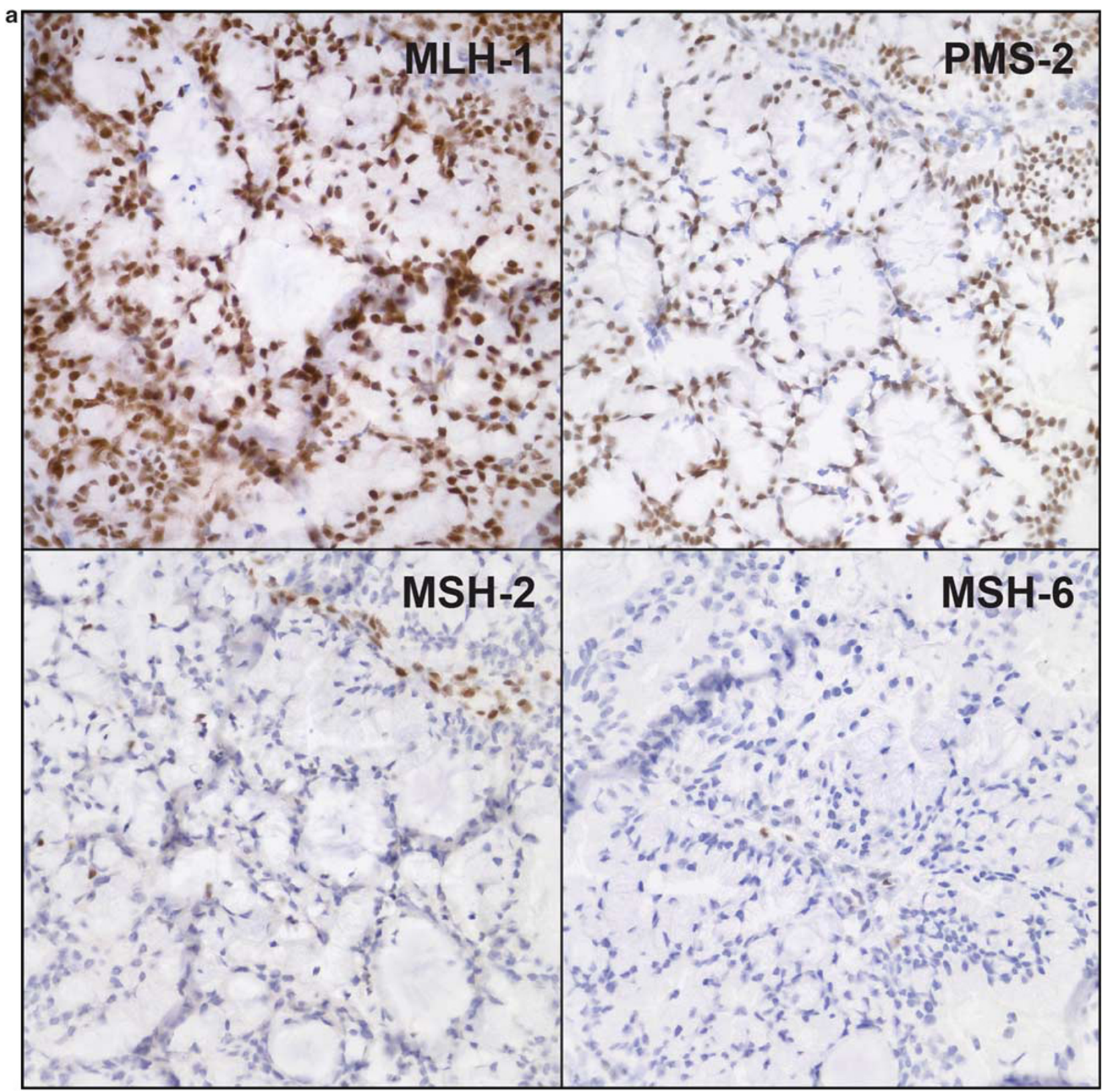

Figure 3 MSI testing by immunohistochemistry (a) and molecular microsatellite analysis (b). Tumor cells have lost normal expression of MSH-2 and MSH-6 proteins. Brown staining of stromal cells is seen (internal control). Expression of $M L H-1$ and $P M S-2$ proteins is intact (positive staining in tumor cells and stromal cells). MSI molecular analysis confirms the presence of high microsatellite instability (MSI-high), as indicated by the presence of non-overlapping allelic peaks of the tumor tissue (b, black and arrowheads) with those of the paired normal endometrium (blue, green, and red) at three of five microsatellite loci. 

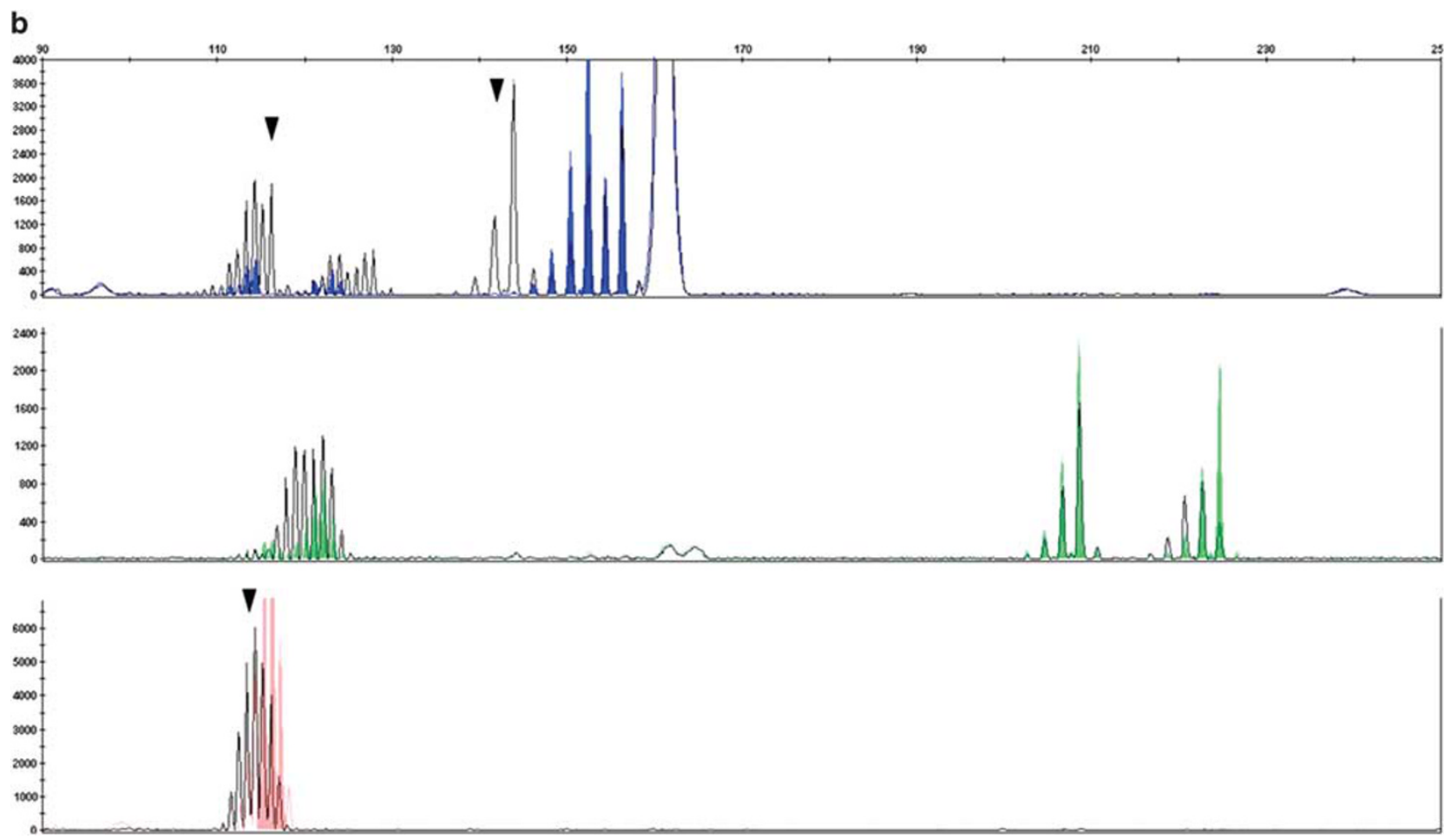

Figure 3 (Continued).

errors of STR genotyping. Nevertheless, recognition of such interference by MSI is feasible as long as a clean dissection of the tissue in question is ensured and the molecular pathologist pays closer attention to the background allelic information, as small allelic sizes matching to those of the paired normal tissue always exist because of the presence of non-neoplastic cells within the tumor (normal fibroblasts, endothelial cells, and various inflammatory cells). Before dismissing the tissue in question as a cancerous floater as a result of identity testing, prompt immunohistochemistry of MMR proteins and/or molecular MSI testing should be performed to rule out an MSI tumor.

Lynch syndrome is characterized by a germline mutation involving one of the DNA mismatch repair genes, leading to microsatellite instability (MSI). The condition manifests clinically with increased cancer risk in various organs, most commonly colorectal cancers. The second most common cancer in Lynch syndrome is endometrial adenocarcinoma with a lifetime risk of around $60 \% \cdot{ }^{7-10}$ In fact, endometrial cancer frequently presents as an index cancer in women with Lynch syndrome. ${ }^{11,12}$ However, the majority of cancers with MSI are caused by somatic methylation of the MLH1 promoter region that silences gene expression in the tumor tissue, rather than germline mutations of MMR genes. Therefore, the presence of MSI in the tumor alone is far from sufficient to confirm Lynch syndrome. Whereas Lynch syndrome accounts for $2 \%$ of all endometrial cancers, MSI was observed in
$34 \%$ of all cases of endometrial carcinoma in one previous study. ${ }^{13}$

In summary, tissue identity testing targeting a cancerous floater can be significantly complicated by the presence of tumor cells that exhibit MSI. Colorectal and endometrial cancers are the two most common tumor types involved by MSI. A high index of suspicion by the molecular pathologist and MSI testing using immunohistochemical and/or molecular methods are crucial to avoid an erroneous conclusion and subsequent clinical and legal consequences.

\section{Disclosure/conflict of interest}

The authors declare no conflict of interest.

\section{References}

1 Hunt JL, Swalsky P, Sasatomi E, et al. A microdissection and molecular genotyping assay to confirm the identity of tissue floaters in paraffin-embedded tissue blocks. Arch Pathol Lab Med 2003;127:213-217.

2 Tsongalis GJ, Berman MM. Application of forensic identity testing in a clinical setting: specimen identification. Diagn Mol Pathol 1997;6:111-114.

3 Lipata F, Parkash V, Talmor M, et al. Precise DNA genotyping diagnosis of hydatidiform mole. Obstet Gynecol 2010;115:784-794.

4 Berg KD, Glaser CL, Thompson RE, et al. Detection of microsatellite instability by fluorescence multiplex polymerase chain reaction. J Mol Diagn 2000;2:20-28. 
5 Zhivotovsky LA, Bennett L, Bowcock AM, et al. Human population expansion and microsatellite variation. Mol Biol Evol 2000;17:757-767.

6 Greenson JK, Bonner JD, Ben-Yzhak O, et al. Phenotype of microsatellite unstable colorectal carcinomas: well-differentiated and focally mucinous tumors and the absence of dirty necrosis correlate with microsatellite instability. Am J Surg Pathol 2003;27:563-570.

7 Meyer LA, Broaddus RR, Lu KH. Endometrial cancer and Lynch syndrome: clinical and pathologic considerations. Cancer Control 2009;16:14-22.

8 Vasen HF, Stormorken A, Menko FH, et al. MSH2 mutation carriers are at higher risk of cancer than MLH1 mutation carriers: a study of hereditary nonpolyposis colorectal cancer families. J Clin Oncol 2001;19:4074-4080.
9 Hampel H, Stephens JA, Pukkala E, et al. Cancer risk in hereditary nonpolyposis colorectal cancer syndrome: later age of onset. Gastroenterology 2005;129:415-421.

10 Stoffel EM, Ford B, Mercado RC, et al. Sharing genetic test results in Lynch syndrome: communication with close and distant relatives. Clin Gastroenterol Hepatol 2008;6:333-338.

$11 \mathrm{Lu} \mathrm{KH}$, Broaddus RR. Gynecologic Cancers in Lynch Syndrome/HNPCC. Fam Cancer 2005;4:249-254.

12 Lanspa SJ, Jenkins JX, Cavalieri RJ, et al. Surveillance in Lynch syndrome: how aggressive? Am J Gastroenterol 1994;89:1978-1980.

13 Matthews KS, Estes JM, Conner MG, et al. Lynch syndrome in women less than 50 years of age with endometrial cancer. Obstet Gynecol 2008;111: 1161-1166. 